\title{
El aprendizaje basado en retos como propuesta para el desarrollo de las competencias clave
}

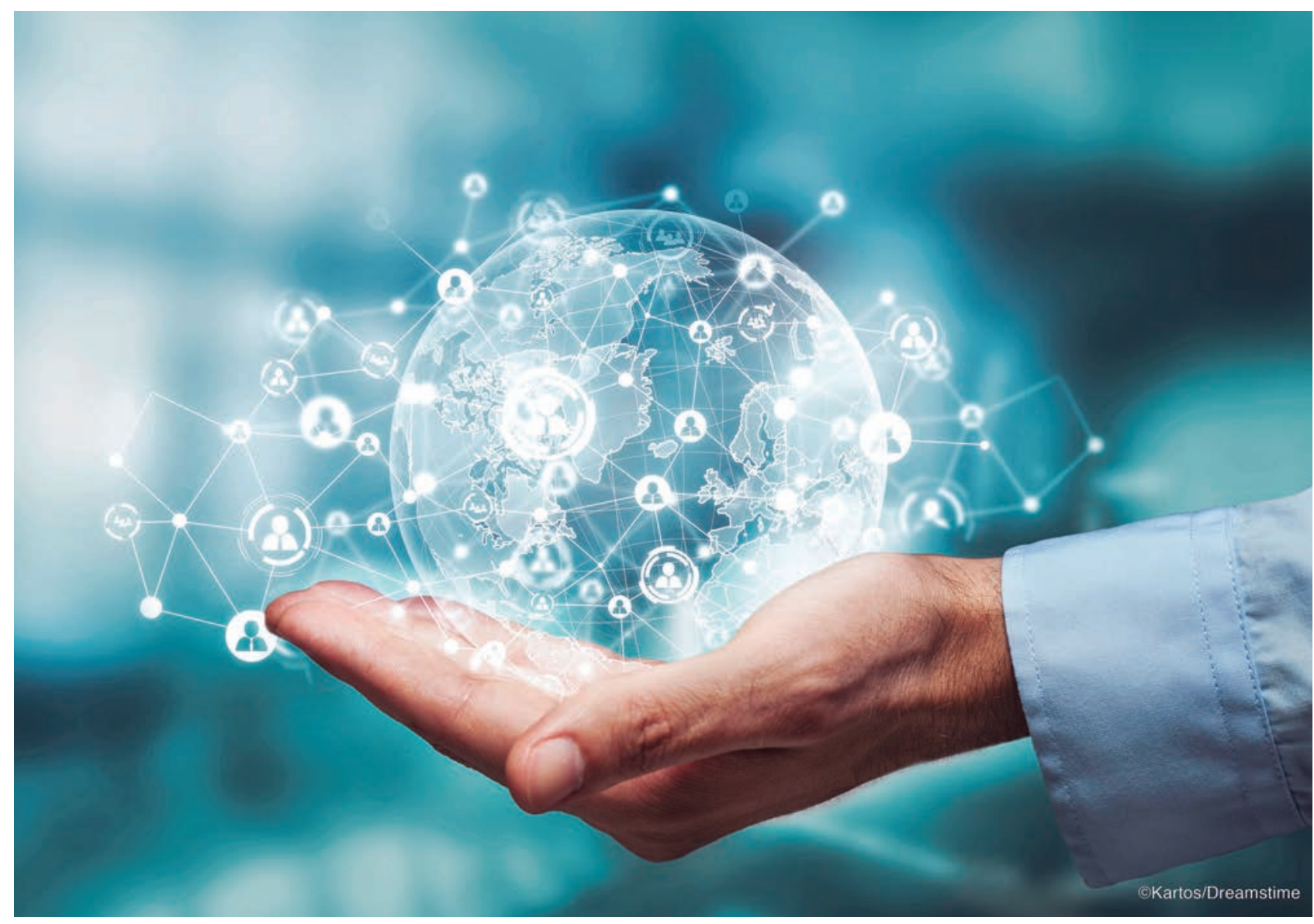

El aprendizaje basado en retos parte de las vivencias y el conocimiento del alumnado de abustosj@gmail.com manera activa y participativa. Este trabajo se centra en la metodología que se puede llevar a cabo en los centros educativos para favorecer este tipo de aprendizaje, así como en su potencial para responder a las exigencias curriculares actuales.

Victoria Castellano Hinojosa victoriacastellanoh@gmail.com

Jacobo Calvo Ramos jacobocalvo@gmail.com

Rafael Mesa Sánchez rafaelmesa2.0@gmail.com

Víctor José Quevedo Blasco vquevedoblasco@cuadernoaula.com

Carmen Aguilar Mendoza teachercarmenn@gmail.com 
La utilización de diferentes metodologías para impulsar el desarrollo de las competencias clave dejó de ser una opción cuando en los currículos oficiales se mostró con mayor detalle el carácter vivencial y funcional de los aprendizajes. En la actualidad, si se pretende dar respuesta a un aprendizaje competencial, los propios elementos curriculares nos muestran en la normativa la necesidad de generar iniciativas didácticas que fomenten la indagación, la experimentación, el trabajo en equipo, la utilización de las tecnologías y las prácticas participativas del alumnado.

Otra de las aportaciones del currículo actual es el de la conexión de los aprendizajes con la vida cotidiana. El conocimiento de la realidad cercana y el desarrollo de propuestas didácticas acordes con ella tienen una clara visibilidad en numerosos contenidos, criterios de evaluación y estándares de aprendizaje. Pero es, sobre todo, en lo relacionado con el desarrollo de las competencias clave donde encontramos las principales aportaciones sobre un aprendizaje contextualizado y coherente respecto a las necesidades de la sociedad actual.

Una mirada a los currículos nos permite observar cómo, de manera prolífica, han aumentado las orientaciones y las sugerencias para la utilización de metodologías activas como idóneas para su desarrollo en los centros educativos. La forma de proceder para llevar a la práctica escolar una correcta concreción de este currículo nos exige actuaciones que estimulen la participación, la iniciativa y el protagonismo del alumnado.

Por otro lado, asistimos de manera recurrente a la aparición en medios de comunicación y redes sociales de propuestas metodológicas que recuperan iniciativas didácticas activas o que bien son adaptadas, en algunos casos incorporando el apoyo visual o tecnológico. Encontramos en este sentido modelos basados en la investigación en el medio, la experimentación, el aprendizaje cooperativo, el aprendizaje-servicio, los centros de interés, los talleres, los estudios

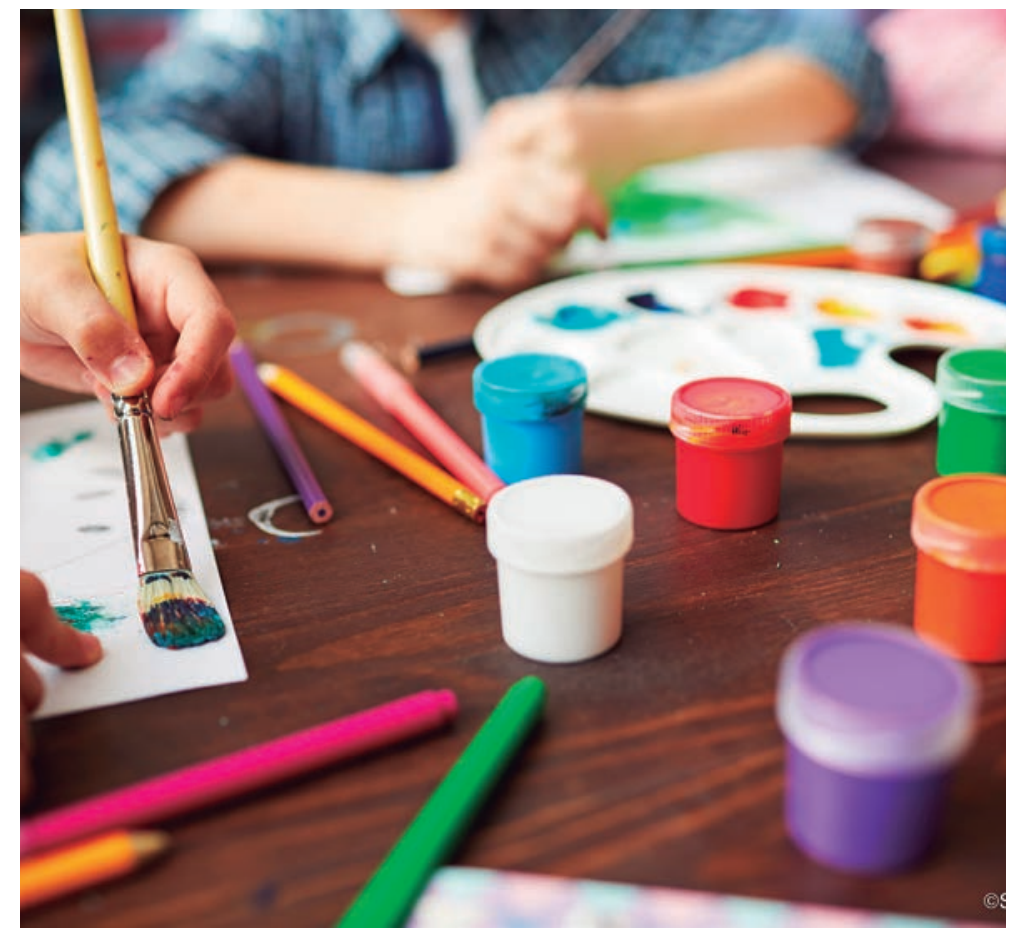

de casos, etc. Generalmente, se conciben y ponen en práctica para la movilización cognitiva del alumnado a través del descubrimiento, el aprendizaje situacional o la manipulación de recursos educativos.

La diversidad de referencias para mostrar alternativas metodológicas es cada vez más notable. Se contribuye así a aumentar las posibilidades de elección para el profesorado, aunque también nos obliga a realizar una permanente revisión sobre sus fundamentos y su aplicabilidad en los centros educativos. Conocer sus potencialidades y dificultades nos permite poner en práctica iniciativas que sean adecuadas desde un enfoque didáctico y también rigurosas respecto a lo que las normas curriculares nos exigen.

En este trabajo nos centraremos en las aportaciones que el aprendizaje basado en retos nos ofrece para el trabajo escolar, Ilevando a cabo una metodología educativa que lo fomente. Para ello, abordaremos aspectos acerca de cómo se fundamenta, en qué consiste y cómo pueden insertarse iniciativas para su desarrollo en la secuencia didáctica.

\section{Conocimiento, vivencia y funcionalidad de los aprendizajes}

El aprendizaje basado en retos nos recuerda por su proximidad epistemológica a planteamientos como el trabajo o aprendizaje por tareas, el aprendizaje basado 


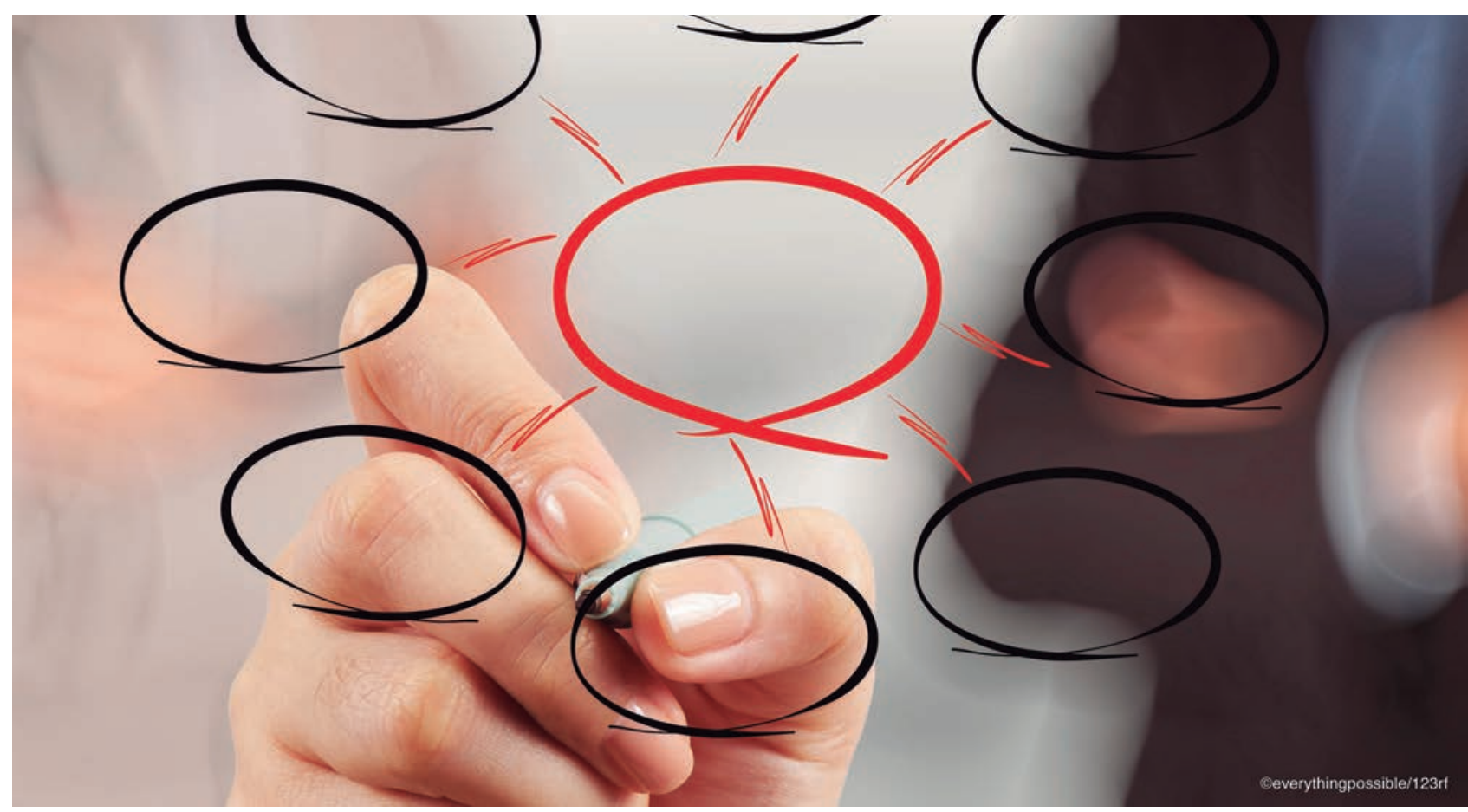

en proyectos, el aprendizaje basado en problemas o los estudios de casos para el contexto didáctico. Todos comparten elementos basados en la construcción de los aprendizajes de manera activa por parte del alumnado, la presentación de situaciones problemáticas sobre las que se generará una solución o producto final, el enriquecimiento del trabajo en torno a propuestas cooperativas del alumnado y un papel más facilitador que protagonista del docente. A través de estos modelos se fomenta la adquisición de logros competenciales por parte del alumnado, priorizando los procesos que intervienen a lo largo del trabajo escolar.

En el ámbito anglosajón encontramos alusiones afines como Challenge Based Learning y Challenge Based Instruction (Johnson, Smith, SMythe, \& VARON, 2009; Observatorio de Innovación Educativa, 2016). Estas propuestas ayudaron a definir un modelo en el que necesariamente se debían presentar respuestas por parte del alumnado a problemas reales de la vida cotidiana, orientando el trabajo hacia la indagación y la aplicación de conocimientos a diversas situaciones.

En el trabajo que aquí presentamos se le otorga al aprendizaje basado en retos la integración del mayor número posible de competencias clave, siempre demandando el dominio de los contenidos curriculares. Los logros se concretan en el éxito para llevar a cabo los procesos para la elaboración de un producto, generalmente realizado de manera cooperativa y que visibiliza de manera tangible el trabajo realizado.

Así pues, el aprendizaje basado en retos es un aprendizaje vivencial en el que se facilita la participación directa y activa de quienes intervienen. El alumnado se siente involucrado desde el comienzo en el trabajo sobre una temática, tomando decisiones, organizando materiales y espacios e indagando en el proceso, por lo que se aportan diferentes estímulos que incentivan la motivación personal y facilitan el aprendizaje. Por tanto, este aprendizaje tratará de dar respuesta a los intereses, a las motivaciones y a las competencias del alumnado (CALvo y MESA, 2018).

\section{El reto como eje del trabajo competencial}

Para comprender mejor el trabajo escolar que fomenta este tipo de aprendizaje es necesario concretar en qué consiste un reto. Suele plantearse tras una situación cercana a las experiencias del alumnado, que sirve como estímulo, y una curiosidad que conecta los aspectos generales de la temática o problema analizado con los específicos que se van a desarrollar a lo largo del trabajo. El reto se presenta formalmente a través de una pregunta que pretende ser provocadora para el alumnado, una 
invitación a la iniciativa. Trata de alentarle en la necesidad de poner en marcha su capacidad de aprendizaje e investigación en torno a los contenidos necesarios para conseguir un producto final.

Por lo tanto, el reto se genera con una intención claramente motivadora. Se crean estímulos cercanos que desencadenan posteriormente actuaciones que facilitan el aprendizaje. De ahí el carácter competencial que tiene su desarrollo, ya que obliga al alumnado a aplicar lo aprendido de manera real y pragmática, realizando, presentando y difundiendo un producto como fruto del trabajo.

Existe una variedad elevada de tipos de productos finales a los que se puede optar en el trabajo escolar de los centros educativos y que pueden tenerse en consideración. Dependiendo de su formato, soporte o forma de difundirlos, podrían serlo exposiciones orales y escritas (con o sin soporte digital), murales, carteles, cómics, periódicos, dosieres, pequeñas investigaciones, maquetas, grabaciones audiovisuales, representaciones teatrales o coreográficas, folletos, blogs, planos y mapas, etc.

Como puede observarse en el ejemplo de reto mostrado en la tabla 1, el alumnado conoce desde el comienzo del trabajo en

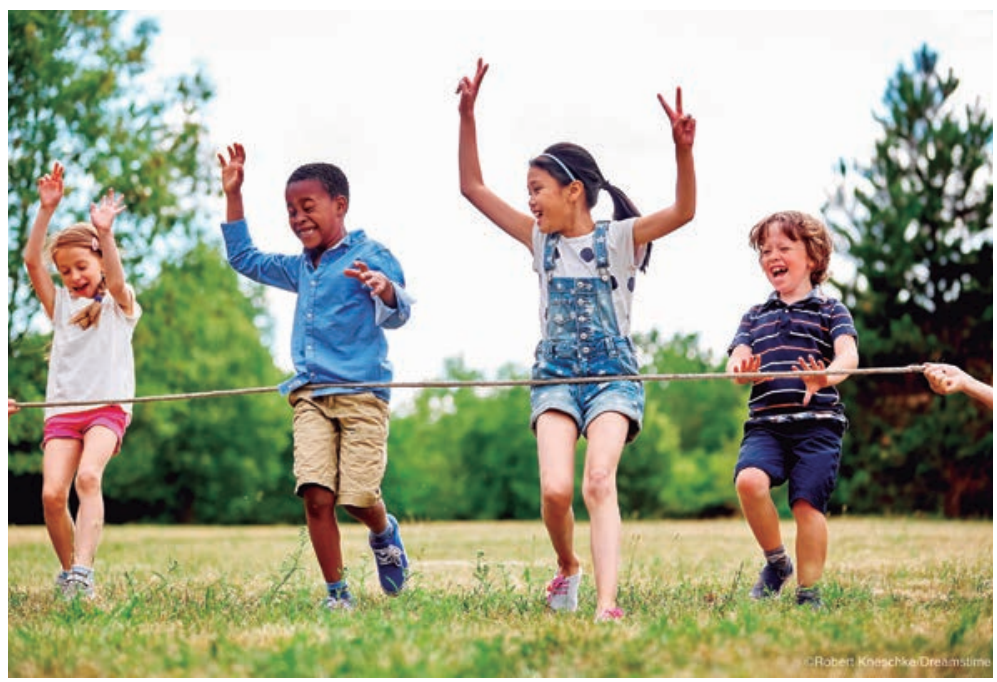

la unidad el planteamiento del trabajo y el producto final al que se optará, con posibilidad de difusión a la finalización del trabajo. Para llevarlo a cabo con éxito, será necesario que su diseño, desarrollo y finalización, a través de un trabajo pautado en diferentes pasos, estén incluidos de manera clara en la secuencia didáctica de cada temática o unidad (aspecto especificado en el apartado "Relación entre los apartados de la unidad y los pasos del reto").

De este modo, el aprendizaje basado en retos pretende dar respuesta a una inquietud o interrogante que se plasmará mediante un producto final, que precisará de aprendizajes (contenidos) y actividades, que se realizarán a lo largo de la unidad o tema propuesto. En este senti-

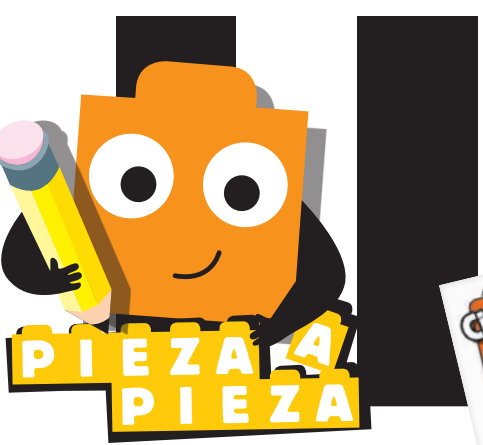

Primaria

Un proyecto basado en el aprendizaje competencial
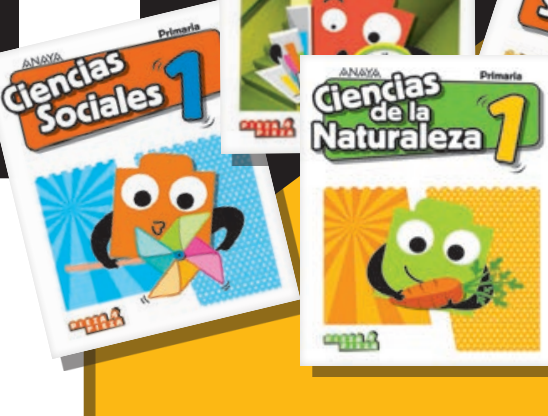

Pieza a Pieza ofrece, con el contrastado rigor curricular de Anaya, un proyecto en el que el profesorado y el alumnado cobran máximo protagonismo a través de una metodología competencial en la que destacan dos elementos básicos: la investigación y aprender haciendo. Un proyecto atractivo, coordinado interdisciplinarmente y secuenciado a lo largo de toda la etapa. 
Tabla 1. Ejemplo extraído de la unidad 5 de Ciencias Sociales 3 (Primaria). Grupo Anaya (2018)

\section{Temática}

Estímulo

\section{Los trabajos y los productos naturales y elaborados}

Ilustración inicial de la unidad en la que se muestra una frutería en la que el padre, la hija y el frutero intercambian palabras en torno a los productos que se venden en este comercio. La escena trata de estimular el análisis y la reflexión en torno a los tipos de trabajo y las actividades económicas

Algunas frutas como las que aparecen comentadas en la ilustración también se pueden transformar en otros productos como, por ejemplo, mermelada. Para poder consumirlos, unas personas los cultivan en el campo; otras los transforman en fábricas y otras los transportan a las tiendas

Situación de partida o curiosidad ¿Te animas a presentar un producto natural o elaborado?

Propuesta de reto

Producto final

Aprendizajes y procesos necesarios para superar el reto

\section{Anuncio publicitario sobre un producto de la zona}

Identificar qué son las materias primas y saber para qué sirven

Conocer y distinguir cuáles son los productos naturales y cuáles los elaborados

Conocer diferentes tipos de trabajo que existen

Relación entre los apartados de la unidad y los pasos del reto Pasos del reto

\section{Apartados}

\section{Los productos naturales y los} productos elaborados

Diferencias entre productos naturales y productos elaborados

\section{Los trabajos en el campo}

Actividades en la agricultura y en la ganadería

\section{Los trabajos en el mar}

Actividades en el mar: la pesca

\section{Los trabajos en talleres y fábricas}

La artesanía y la industria

\section{Los trabajos en el comercio}

Actividades en el transporte y en el comercio
1. Decidir el producto que se va a anunciar. En gran grupo se elige un producto que se obtiene 0 produce en la zona

2. Decidir cómo será el anuncio y cómo organizarse para realizarlo. Se traen anuncios de casa, en revistas, periódicos, folletos, etc., y se dan a conocer en clase. Se decide cuál servirá de modelo para anunciar el producto elegido y se distribuyen las tareas: redactar el título, los textos, buscar imágenes, etc.

3. Recoger y redactar las características más importantes del producto. En un borrador se escriben las características especiales del producto. Puede ser de utilidad saber cómo se obtiene y por cuánto dinero se suele vender. Para ello se puede consultar a alguien que lo fabrique o que lo venda

4. Elegir una imagen y un título que represente el producto. Cada grupo aglutinará la información obtenida, la analizará y la expondrá al resto de la clase, con la posibilidad de publicarse en algún medio del centro

5. Realizar el anuncio y presentarlo. Se pasa a limpio, revisando la ortografía y cuidando la presentación, y se presenta al resto de la clase. Se puede elegir uno para la clase o realizar uno común para que se publique en el periódico del colegio, en el blog o en la web do, la metodología para llevar a cabo este aprendizaje podrá tener visibilidad en los documentos de los centros educativos y en la planificación formal del trabajo escolar. Las actividades asociadas al desarrollo del reto han de quedar reflejadas en cada apartado o sección como aportaciones enriquecidas competencialmente.

Gran parte de los aprendizajes asociados a los contenidos será necesario transferirlos a la realización del reto, con lo que estaremos favoreciendo la generación de procesos cognitivos de orden superior y la implementación de competencias clave. Lógicamente, cada uno de los apartados de la unidad contendría otras actividades que ayudarían en la adquisición y aprendizaje de los contenidos.

Así, un reto no podrá considerarse una propuesta fruto de la acumulación de ac- tividades descontextualizadas de la realidad temática en estudio. Por el contrario, mantendrá siempre la conexión con la naturaleza de los contenidos y con las experiencias familiares, sociales y culturales próximas al alumnado.

Por otro lado, ante los procesos de cooperación necesarios entre el alumnado para la realización de la mayor parte de los retos son esperables, lógicamente, el desarrollo de habilidades interpersonales, la mejora de las competencias emocionales o la estimulación de la creatividad y la capacidad para emprender.

\section{La presencia del currículo}

Una de las dudas que en ocasiones surge en torno a la implementación de las metodologías activas es la de si fomentan con rigor la aplicación y el desarrollo de 
un currículo cada vez más estructurado. El profesorado que se siente atraído por ellas necesita contar con la seguridad de cumplir con lo que la normativa nos demanda.

Las propuestas metodológicas sin el soporte curricular podrían ser iniciativas que involucran al alumnado y favorecen el desarrollo de aspectos personales, afectivos o sociales; propuestas loables, pero alejadas del cumplimiento de la norma. Llevar a cabo iniciativas de este tipo, aparentemente innovadoras en lo relacionado con la práctica escolar, puede tener sin duda cierto interés pedagógico y, sin embargo, incumplir con lo que determina el corpus legal concebido, diseñado y publicado para cada territorio.

Si nos apartamos de lo que los currículos plantean, dar respuesta a modelos metodológicos que tratan de involucrar al alumnado, fomentar la cooperación o que abordan temáticas comunes y compartidas en la elaboración de productos competenciales formarían parte de un conjunto de acciones descontextualizadas de las propuestas del currículo establecido. En esencia, las aportaciones prescriptivas que en la legislación existen son el marco de referencia para cualquier actuación educativa rigurosa en las enseñanzas regladas, independientemente de la opción metodológica utilizada. En el caso de algunas administraciones, y como ayuda nada despreciable, observamos durante los últimos años en sus currículos oficiales planteamientos y ejemplificaciones que fomentan de una manera más abierta el uso de metodologías flexibles.

\section{ÁQORA DE DROFESORES}

Te servirán de ayuda para llevar a cabo propuestas basadas en retos las siguientes orientaciones (Calvo y Mesa, 2018):

1. El planteamiento del reto lleva implícita la realización de un producto final para el que se pondrán en juego contenidos y competencias clave.

2. El producto final se generará a lo largo de varios pasos.

3. Los pasos tendrán relación temática con algunos apartados 0 secciones de las unidades, temas 0 bloques disciplinares.

4. Se deberá dominar parte de los contenidos de cada apartado o sección para realizar con éxito cada paso. Para ello, cada paso se insertará en el espacio de desarrollo de cada apartado o sección temática.

5. Se tratará de que la parte final del reto incluya una difusión en algún canal interno o externo del centro educativo. Podrá ser dentro del propio grupo, a las familias, a la comunidad educativa e incluso al ámbito social inmediato (instituciones 10cales, asociaciones...), próximo (entidades provinciales, regionales...) 0 lejano (entidades nacionales o internacionales).

6. Por su carácter interdisciplinar, podrá tener presencia de aportaciones de otras áreas o materias de aprendizaje.

Así pues, la forma en que el currículo oficial se integra en las propuestas de trabajo escolar, o cómo las metodologías lo respetan y lo desarrollan, será una constante en la planificación de actuaciones educativas en torno a opciones como el aprendizaje basado en retos. Los productos finales obtenidos tras la superación de cada reto, así como los procesos llevados a cabo para su obtención y difusión, deberán estar en consonancia con los elementos curriculares establecidos para cada nivel y área. Los objetivos de los que de manera intencional se parte en el desarrollo de los retos y, sobre todo, los contenidos involucrados en la realización de los pasos necesarios para la generación de productos finales, ayudarán a fomentar la adquisición de competencias clave •

\section{(D.) DARA SABER MÁS}

Calvo, L. J., y Mesa, R. (2018). Pieza a pieza. Las claves del proyecto. Madrid: Grupo Anaya.

Johnson, L. F., Smith, R. S., Smythe, J. T., y Varon, R. K. (2009). Challenge-Based Learning: An Approach for Our Time. Austin, Texas: The New Media Consortium.

Observatorio de Innovación Educativa. (2016). Edu Trends. Aprendizaje basado en retos. Moterrey-Nuevo León (México): Editorial Instituto Tecnológico y de Estudios Superiores de Monterrey. 